\title{
TEMÁTICA DOS ARTIGOS DE PERIÓDICOS BRASILEIROS NAÁREA DA CIÊNCIA DA INFORMAÇÃO NA DÉCADA DE 90
}

\section{THE THEMESOFTHEARTICLES THE BRAZILIANSCIENTIFICPERIODICALS IN THE INFORMATION SCIENCE AREA IN THE DECADE OF 90}

\author{
Cláudia Maria Pinho de Abreu PECEGUEIRO'
}

\section{RESUMO}

\begin{abstract}
Considerando-se a importância da comunicação científica para o progresso da ciência como forma de divulgar e patentear a pesquisa científica, analisa-se o periódico científico, impresso e eletrônico na área da Ciência da Informação. Objetiva-se obter um panorama dos temas publicados nos periódicos científicos brasileiros na área de Ciência da Informação na década de 90 visando identificar as tendências temáticas nacionais, a relação dos temas brasileiros com os internacionais e a existência de grupos de pesquisadores de acordo com suas publicações nesses periódicos brasileiros.
\end{abstract}

Palavras-Chave: periódicos científicos, ciência da informação, artigos científicos, produtividade de autores.

\section{ABSTRACT}

Analysis of the printed and electronic scientific periodical in the Information Science area; considering the importance of scientific communication for the progress of science, as a way of spreading and patenting scientific research. The target is to get a panorama of published themes in Brazilian scientific periodicals in the Information Science area in the 90s; aiming at identifying the thematic trends in Brazil, the relation between Brazilian themes and international themes and the existence of researchers according to their publications in these Brazilian periodicals.

Key words: scientific periodicals - Brazil, information science, periodical articles, subjects.

\section{INTRODUÇÃO}

A comunicação é uma atividade inerente ao homem, o que o diferencia a partir da linguagem, de outros animais. A produção científica, formalização da comunicação científica, torna-se assim objeto de circulação de idéias. A divulgação dos resultados e andamentos das pesquisas é feita preferencial-

\footnotetext{
(1) Professora do Departamento de Biblioteconomia, Universidade Federal do Maranhão. E-mail: clpecegueiro@uol.com.br
} 
mente nos periódicos científicos, fonte primária considerada de maior importância para a comunidade científica por ser um canal ágil na disseminação de novos conhecimentos.

O estudo do desenvolvimento da produção científica e das formas nas quais essa se torna pública e é assimilada pela comunidade científica vem sendo efetuado pelos cientistas da informação ao longo do tempo. No Brasil os trabalhos de Dumont et al. (1979); Neves \& Melo (1980); Miranda (1981); Forest \& Martins (1987); Foresti (1990); Pitella (1991); Araújo \& Cunha (2000); e Ohira (2000), entre outros, estudaram a produção científica na área da Ciência da Informação e Biblioteconomia, avaliando o comportamento da literatura veiculada nos periódicos científicos, teses e dissertações.

Seguindo essa linha, esse estudo tem por objetivo estudar os periódicos brasileiros na área da Ciência da Informação na década de 90, procurando identificar, a partir de seus artigos, as tendências gerais da literatura, produtividade dos autores, autoria única e em colaboração, volume dos artigos e idiomas.

\section{MATERIALE MÉTODOS}

O universo é compreendido pelos artigos de seis periódicos brasileiros na área de Ciência da Informação e Biblioteconomia: Ciência da Informação (Ci. Inf.), Informação \& Sociedade: estudos (Inf. Soc.), Perspectivas em Ciência da Informação (Pers.), Revista da Escola de Biblioteconomia da UFMG (Rev. UFMG), Revista de Biblioteconomia de Brasília (R.B.B.),
Transinformação (Trans.), demonstrando a produção de artigos por título e fascículo no período (Tabela 1). Cabe ressaltar que nesta pesquisa não foram consideradas as seções denominadas "Temas em debate", "Comunicação de Pesquisa" entre outras. Para seleção da amostra, foram excluídos os periódicos que não possuem número de série International Standard Serial Number (ISSN) e os periódicos que não publicaram no período em questão, década de 90 com a média mínima de 5 artigos por fascículos conforme critérios do Programa de Apoio a Publicações Científicas mantido pelo Conselho Nacional de Desenvolvimento Científico e Tecnológico (CNPq), Ministério de Ciência e Tecnologia.

A amostra foi composta, então, por seis periódicos, perfazendo um total de 627 artigos (Tabela 1), selecionados com base nos parâmetros já descritos. A Revista da Escola de Biblioteconomia da UFMG deixou de ser editada em 1995. No lugar dela surgiu a Revista Perspectivas em Ciência da Informação, editada pela mesma Escola. Apesar da nota, nas revistas, ligando os dois títulos, eles são tratados separadamente neste estudo, pois, além das mudanças de título e apresentação, têm, também ISSN diferentes.

\section{RESULTADOSEDISCUSSÃO}

\section{Periódicos}

Observou-se as seguintes características, título do periódico, local de publicação, editor (entidade responsável), data (ano do primeiro

Tabela 1. Distribuição de artigos, por periódico (1990-1999).

\begin{tabular}{lcccc}
\hline Periódico & Artigos publicados (n) & $\%$ & Fascículos & Média Artigo/Fascículo \\
\hline Ciência da Informação & 248 & 39,55 & 25 & 9,92 \\
Informação e Sociedade & 58 & 9,25 & 9 & 6,63 \\
Perspectivas em Ciência da Informação & 60 & 9,57 & 8 & 7,50 \\
Revista da UFMG & 87 & 13,88 & 13 & 6,77 \\
Revista de Biblioteconomia de Brasília & 73 & 11,64 & 10 & 7,30 \\
Transinformação & 101 & 16,11 & 33 & 3,06 \\
\hline Total & 627 & 100,00 & 98 & 6,39 \\
\hline
\end{tabular}


volume e, se a publicação cessou, também do último), periodicidade e ISSN, consideradas elementos essenciais e complementares à identificação do periódico (Tabela 2).

A Região Sudeste concentra o maior número de instituições responsáveis/editoras que publicam os periódicos científicos, além de um maior número de Cursos de Pós-Graduação na área. Neves \& Melo (1980) afirmam que profissionais de outras regiões encontram dificuldades em publicar nestes periódicos. Mueller (1996) considera que os artigos publicados são principalmente de autoria de professores dos cursos ligados diretamente ao órgão responsável pela publicação dos periódicos. Acredita-se que se hoje o local da edição exerce influência no que se refere à autoria dos artigos, isso tende a ser minimizado. A exemplo, pode-se citar políticas de Pós-Graduação que visam a difusão do conhecimento através dos cursos de Mestrado/Doutorado interinstitucional, além do uso da Internet que quebra barreiras geográficas.

Quanto ao editor, as Revistas seguem tendências já levantadas em registros anteriores Miranda (1981); Foreste \& Martins (1987); Mueller (1992); Mueller et al. (1996), e estão ligadas principalmente às instituições com cursos de Pós-Graduação em Ciência da Informação e Biblioteconomia e aos órgãos profissionais. Os cursos, além de concentrarem um maior número de pesquisas na área, são também disseminadores de suas pesquisas.

A década de 70 pode ser considerada como um marco na área da Ciência da Informação no Brasil. Nesta década foram criados seis cursos de mestrado, o que reflete o interesse do governo em apoiar a educação no âmbito da Pós-Graduação. Esta política incentivou a produção científica na área que encontra nos periódicos um veículo de divulgação dos resultados dos estudos. Nesse período originam-se os periódicos Ciência da Informação, Revista de Biblioteconomia da UFMG e Revista de Biblioteconomia de Brasília e na década de 90, a Transinformação, Perspectivas em Ciência da Informação e Informação \& Sociedade: estudos.

Os seis títulos estudados apresentam periodicidade equivalente. A maioria é semestral: Revista da Escola de Biblioteconomia da UFMG, Revista de Biblioteconomia de Brasília e Perspectivas em Ciência da Informação. A Revista Ciência da Informação foi semestral até o ano de 1991, quando assumiu periodicidade trimestral. Somente o periódico Informação \& Sociedade: estudos tem, no período da pesquisa, periodicidade anual. A periodicidade, elemento que garante a credibilidade do periódico impresso, parece ser difícil de ser mantida. Isso pode ser caracterizado pela falta de financiamento constatada por Miranda (1981, p. 36) o qual afirma que "O financiamento é, talvez, um dos problemas mais sérios porque as revistas não são autofinanciáveis".

OISSN, identificador que individualiza o título da publicação seriada, está presente em todos os periódicos, o que possibilita rapidez e precisão na identificação e controle dessas publicações.

\section{Temas}

A classificação dos artigos por tema foi realizada com base na Tabela de Assuntos dos

Tabela 2. Identificação dos periódicos.

\begin{tabular}{|c|c|c|c|c|c|}
\hline Título do Periódico & Local de Publicação & Editor & Data & Periodicidade & ISSN \\
\hline Ciência da Informação & Brasília & IBICT & 1972 & Quadrimestral & $0100-1965$ \\
\hline Informação e Sociedade & João Pessoa & UFPB/Dep. BIB & 1991 & Anual & 0104-0146 \\
\hline Perspectivas em Ciência da Informação & Belo Horizonte & Esc. Biblioteca UFMG & 1996 & Semestral & $1413-9936$ \\
\hline Revista UFMG & Belo Horizonte & Esc. Biblioteca UFMG & $1972-1995$ & Semestral & 0100-0829 \\
\hline Revista de Biblioteconomia de Brasília & Brasília & ABDF e UnB/CID & 1973 & Semestral & $0100-7157$ \\
\hline Transinformação & Campinas & PUCCAMP/BIB & 1992 & Quadrimestral & 0103-3786 \\
\hline
\end{tabular}


Periódicos na Área da Ciência da Informação, proposta por Teixeira (1997), com algumas adaptações. Para esse trabalho, utilizou-se as dez classes principais, grafadas em negrito. As subdivisões dessas classes foram utilizadas apenas para facilitar a classificação (Anexo 1).

Foi observada a freqüência dos temas por classe e a freqüência de 1990 a 1999. A predominância do tema 8: Entrada, Tratamento, Armazenamento, Recuperação e Disseminação da Informação com o total geral de 131 ocorrências (20,9\%); seguido dos temas 4: Estudos de Usuário, Transferência da Informação e Uso da Biblioteca com o total de 128 ocorrências $(20,4 \%)$; e do tema 3: Organização e Gerência de Atividades de Informação, de Bibliotecas e Centros de Pesquisa com o total de 127 ocorrências (20,3\%). O grau de ocorrência dos temas restantes sempre se encontra abaixo de 11,0\% (Tabela 3). Não houve artigos sobre o tema 6: Prédios de Biblioteca. A ênfase dada aos assuntos referentes à informação (temas 3,4 e 8) se manteve, talvez, pelo uso das novas tecnologias de comunicação. Estas três áreas em conjunto são responsáveis por mais de $60,0 \%$ do total de artigos (Dumont et al. (1979); Neves \& Melo (1980); Miranda (1981).

Na Base de Dados LISA (Tabela 4), foi verificada uma frequiência bem diferente. Há uma predominância de artigos referentes ao tema 2: Ensino, Atividade Profissional e Pesquisa que totalizam 7507 artigos (70,8\%), dos quais 7492 concentram-se na área de pesquisa, seguido do tema 6: Prédios de Biblioteca, cujo número de ocorrências totaliza 1083 artigos (10,2\%). Os temas restantes não atingem, no total, uma porcentagem superior a $19,0 \%$.

Para efeitos comparativos, considerou-se o total de ocorrência dos temas nos periódicos nacionais (627) e dos temas indexados pelo LISA (10603). Lançou-se mão do uso da percentagem para compararmos o grau de importância de cada tema dentro da sua referida amostra. Dos resultados, pode-se inferir os seguintes comentários: No que se refere à freqüência dos temas, o tema 1: Ciência da Informação, Biblioteconomia e Documentação (generalidades), tema 3: Organização e Gerência de Atividades de Informação de Bibliotecas e Centros de Pesquisa, tema 4: Estudo

Tabela 3. Distribuição de temas por periódicos.

\begin{tabular}{|c|c|c|c|c|c|c|c|c|}
\hline \multirow{3}{*}{ Tema } & \multicolumn{6}{|c|}{ Periódicos } & \multirow{2}{*}{\multicolumn{2}{|c|}{ Total }} \\
\hline & \multirow{2}{*}{$\begin{array}{l}\text { Ciência da } \\
\text { Informação }\end{array}$} & \multirow[t]{2}{*}{ Perspectivas } & \multirow{2}{*}{$\begin{array}{c}\text { Revista de } \\
\text { Biblioteconomia de } \\
\text { Brasília } \\
\end{array}$} & \multirow[t]{2}{*}{$\begin{array}{l}\text { Revista da } \\
\text { UFMG }\end{array}$} & \multirow[t]{2}{*}{ Transinformação } & \multirow[t]{2}{*}{$\begin{array}{c}\text { Informação e } \\
\text { Sociedade }\end{array}$} & & \\
\hline & & & & & & & $\mathrm{n}$ & $\%$ \\
\hline 1 & 22 & 8 & 4 & 6 & 7 & 7 & 54 & 8,6 \\
\hline 2 & 8 & 5 & 9 & 13 & 21 & 10 & 66 & 10,5 \\
\hline 3 & 49 & 6 & 27 & 27 & 12 & 6 & 127 & 20,3 \\
\hline 4 & 53 & 13 & 7 & 16 & 24 & 15 & 128 & 20,4 \\
\hline 5 & 26 & 2 & 6 & 4 & 12 & 0 & 50 & 8,0 \\
\hline 6 & 0 & 0 & 0 & 0 & 0 & 0 & 0 & 0,0 \\
\hline 7 & 4 & 4 & 2 & 3 & 1 & 0 & 14 & 2,2 \\
\hline 8 & 64 & 15 & 17 & 13 & 17 & 5 & 131 & 20,9 \\
\hline 9 & 13 & 4 & 1 & 2 & 2 & 5 & 27 & 4,3 \\
\hline 10 & 9 & 3 & 0 & 3 & 5 & 10 & 30 & 4,8 \\
\hline Total & 248 & 60 & 73 & 87 & 101 & 58 & 627 & 100,0 \\
\hline
\end{tabular}


de Usuário, Transferência e Uso da Informação e Uso da Biblioteca, tema 5: Estudo da Literatura e do Documento, tema 8: Entrada, Tratamento, Armazenamento, Recuperação e Disseminação da Informação e tema 9: Política de Informação e Política Científica e Tecnológica são mais representados nos periódicos nacionais que os encontrados no LISA. Por outro lado, os temas 2: Ensino, Atividade Profissional e Pesquisa, tema 6: Prédios de Biblioteca e tema 7: Serviços Técnicos estão mais presentes nas agendas de pesquisa em âmbito internacional.

A soma de autores, conforme os temas publicados, totaliza 785 autores. Isso se deve a um mesmo autor publicar artigos em dois ou mais temas. Sendo assim, verificou-se a maior concentração de autores (167) no tema 3: Organização e Gerência de Atividades de Informação, de Bibliotecas e Centros de Pesquisa, seguido do tema 8: Entrada, Tratamento, Armazenamento, Recuperação e Disseminação da Informação com 164 autores e do tema 4: Estudos de Usuário, Transferência da Informação e Uso da Biblioteca, com o total de 158 autores. Os demais temas totalizaram 269 autores e não chegam a atingir individualmente um percentual maior que $11 \%$.

A distribuição de temas por ano de publicação chama a atenção à oscilação de artigos sobre determinados temas em alguns anos (Tabela 5). O

Tabela 4. Distribuição de temas no LISA.

\begin{tabular}{crc}
\hline \multirow{2}{*}{ Tema } & \multicolumn{2}{c}{ LISA } \\
\cline { 2 - 3 } 1 & 380 & $\%$ \\
\hline & 15 & 3,58 \\
2 & 7492 & \\
3 & 7 & 70,80 \\
& 45 & 0,07 \\
4 & 24 & \\
5 & 10 & 0,66 \\
6 & 1083 & 0,09 \\
7 & 733 & 6,91 \\
8 & 34 & 0,33 \\
9 & 780 & 7,35 \\
10 & - & - \\
\hline Total & 10603 & 100,00 \\
\hline
\end{tabular}

Fonte: Base de dados LISA.

Tabela 5. Distribuição de temas por ano de publicação.

\begin{tabular}{|c|c|c|c|c|c|c|c|c|c|c|c|}
\hline \multirow{2}{*}{ Temas } & \multicolumn{11}{|c|}{ Anos } \\
\hline & 1990 & 1991 & 1992 & 1993 & 1994 & 1995 & 1996 & 1997 & 1998 & 1999 & Total \\
\hline $\mathrm{T} 1$ & 6 & 3 & 5 & 3 & 2 & 8 & 9 & 3 & 7 & 8 & 54 \\
\hline $\mathrm{T} 2$ & 17 & 5 & 2 & 4 & 5 & 7 & 10 & 4 & 7 & 5 & 66 \\
\hline T3 & 29 & 8 & 6 & 10 & 14 & 11 & 14 & 10 & 11 & 14 & 127 \\
\hline $\mathrm{T} 4$ & 13 & 10 & 9 & 12 & 9 & 15 & 17 & 23 & 11 & 9 & 128 \\
\hline T5 & 3 & 5 & 4 & 1 & 3 & 2 & 11 & 8 & 9 & 4 & 50 \\
\hline T6 & 0 & 0 & 0 & 0 & 0 & 0 & 0 & 0 & 0 & 0 & 0 \\
\hline $\mathrm{T} 7$ & 3 & 2 & 2 & 2 & 1 & 0 & 2 & 2 & 0 & 0 & 14 \\
\hline T8 & 12 & 10 & 13 & 8 & 13 & 14 & 14 & 19 & 14 & 14 & 131 \\
\hline T9 & 1 & 4 & 3 & 5 & 0 & 1 & 1 & 2 & 1 & 9 & 27 \\
\hline $\mathrm{T} 10$ & 2 & 0 & 1 & 2 & 4 & 7 & 9 & 0 & 0 & 5 & 30 \\
\hline Total & 86 & 47 & 45 & 47 & 51 & 65 & 87 & 71 & 60 & 68 & 627 \\
\hline
\end{tabular}

Fonte: Revistas Ciência da Informação; Informação e Sociedade; Perspectivas em Ciência da Informação, Revista de Biblioteconomia de Brasília, Revista da UFMG, Transinformação (1990-1999).

T1= Ciência da Informação, Biblioteconomia e Documentação; T2= Ensino, Atividades. Prof. e Pesquisa; T3= Organização, Gerência de Atividades Informação; T4= Estudo de Usuário, Transferência, Uso da Informação e Uso da Biblioteca; T5= Est. da Lit. e Doc.; T6= Prédio de Bibliotecas; T7= Serviço Técnico; T8= Entrada, Tratamento, Armazenamento, Recuperação e DSI; T9 = Pol. Informação C\&T; $\quad$ T10 = Outros. 
tema 2: Ensino, Atividade Profissional e Pesquisa apresenta pico de 17 artigos no ano de 1990 e somente dois artigos no ano de 1992; da mesma forma o tema 3: Organização e Gerência de Atividades de Informação, de Bibliotecas e Centros de Pesquisa aparece com o máximo de 29 artigos, em 1990 e o mínimo em 1992 com apenas 6 artigos. O tema 8: - Entrada, Tratamento, Armazenamento, Recuperação e Disseminação da Informação, parece manter uma constância em relação ao ano. A inexistência de artigos sobre o tema 6: Prédios de Biblioteca indica uma falta de interesse que reflete em falta de investimento na construção e ambientação de bibliotecas que cede espaço às bibliotecas virtuais e digitais da década de 90 . Sobre o tema 7: Serviços Técnicos, muito pouco foi publicado no período, 14 ocorrências no total. Nos anos de 1995, 1998 e 1999 não houve artigo publicado sobre o tema.

A concentração de artigos sobre o mesmo tema por ano, a exemplo dos temas 2 e 3 em 1990, sugere uma tendência de interesse da época em estudar determinado assunto, ocasionando fascículos temáticos, ou quase temáticos dos periódicos estudados.

\section{Autores}

Dumont et al. (1979); Neves \& Melo (1980); Miranda (1981); Guimarães (1984); Foresti \& Martins (1987) constataram que a autoria única predomina sobre autoria múltipla ou em colaboração. Estudos de autores únicos parecem ser também uma prática da Ciência da Informação, na América Latina e Caribe (Arenas et al., 2000). Na amostragem deste estudo foi verificado que dos 627 artigos analisados, $461(73,52 \%)$ foram de autoria única e somente $166(26,48 \%)$ foram escritos por mais de um autor.

Isso acontece em razão do próprio subdesenvolvimento da pesquisa na área, que está quase sempre vinculada a trabalhos de autores cursando a Pós-Graduação ou em preparação de trabalhos para apresentação em Congressos ou atividades docentes, sendo a pesquisa institucional, grupal e com vinculação a projetos mais estruturados quase sempre uma exceção, e não a regra(GUIMARÃES, 1994,p. 38).

O periódico Transinformação foi o que apresentou o maior número de artigos em

Tabela 6. Distribuição da autoria - produtividade de autor (1990-1999).

\begin{tabular}{ccccc}
\hline Autores (n) & Artigos (n) & $\%$ & Autor x Artigos & $\%$ \\
\hline 469 & 1 & 75,52 & 469 & 50,48 \\
\hline 82 & 2 & 13,20 & 164 & \} \\
35 & 3 & 5,64 & 105 & \\
14 & 4 & 2,25 & 56 & \\
7 & 5 & 1,13 & 35 & \\
7 & 6 & 1,13 & 42 & \\
3 & 7 & 0,48 & 21 & \\
0 & 8 & 0 & 0 & \\
3 & 9 & 0,48 & 27 & \\
1 & 10 & 0,16 & 10 & \\
\hline 621 & & 100,00 & $929 *$ & \\
\hline
\end{tabular}

Fonte: Revistas Ciência da Informação, Informação e Sociedade, Perspectivas em Ciência da Informação, Revista de Biblioteconomia de Brasília, Revista da UFMG, Transinformação.

(*) Autores e artigos foram computados mais de uma vez, no caso de autoria múltipla.

Média de artigo por autor: Total $/$ artigo $=929 ; \quad$ Total $/$ autor $=621 ;$ Média $=1,49$ 
colaboração na década de $90(33,66 \%)$. O menor índice ficou com o periódico Informação \& Sociedade: Estudos com 18,97\%. Não parece haver nenhuma relação entre o periódico e a autoria individual ou coletiva. Embora não fora aprofundado, pode-se afirmar que os periódicos não fazem recomendações em relação à autoria.

Com relação à produtividade, a média do período estudado nessa pesquisa foi de 1,49 artigos por autores. Trabalhando com um universo de 929 artigos, computando-se mais de uma vez o mesmo artigo nos casos de autoria múltipla, verifica-se que num total de 621 autores, a maioria (469), em um percentual de $75,52 \%$, publicou apenas um artigo e somente um autor publicou 10 artigos, que representam $0,16 \%$ (Tabela 6 ).

A distribuição de autores por periódico demonstra grande concentração no periódico Ciência da Informação com um total de 272 autores $(37,21 \%)$, isto se for considerado o mesmo autor mais de uma vez. Os demais periódicos não ultrapassam individualmente a $18,00 \%$ no total. Considerada de excelência, a Revista Ciência da Informação sempre contou com o apoio institucional do IBICT que lhe garantiu publicação ininterrupta e, consequientemente, um maior interesse dos autores em publicar neste periódico.

A relação de autores que publicaram em mais de um periódico (Anexo 2) teve neste estudo pouca expressividade. De um total de 621 autores, apenas $82(13,2 \%)$ publicaram artigos em mais de um periódico. A maioria dos autores, 538, publicou apenas em um periódico. Cinqüenta e nove autores publicaram em dois periódicos, 21 publicaram em três periódicos. 2 autores publicaram em quatro periódicos e somente 1 autor publicou em cinco periódicos; nenhum autor publicou nos seis periódicos. A penetração dos autores nos diferentes periódicos nesta área, já havia sido estudada por Neves \& Melo (198, p. 426) que concluíram que "O inter-relacionamento dos colaboradores das revistas é quase nulo. Cada uma tem o seu corpo de autores, composto, em sua maioria, de pessoas ligadas, de alguma maneira, à instituição editora".

A análise da distribuição de páginas por artigos de periódicos demonstra não haver, por parte do periódico, nenhuma recomendação. Artigos entre 10 e 20 páginas são os mais freqüentes. A revista que apresentou o maior número de páginas por artigo, durante a década de 90 , foi a Revista de Biblioteconomia de Brasília, com 54 páginas, de autoria de Luiz Renato Vieira, que trata da editoração no Brasil (Tema 3). Com o menor número de páginas ficaram as revistas Ciência da Informação e Informação \& Sociedade: Estudos com artigos de apenas 2 páginas cada um.

\section{CONSIDERAÇÕES FINAIS}

Quanto aos periódicos, conforme preconiza Mueller et al. (1996, p. 350), os periódicos na área da Ciência da Informação no Brasil "poderiam ser considerados suficientes, se todos se mantivessem em dia". O que observou nesta pesquisa foi que além da dificuldade de manter a periodicidade, há ainda uma grande "morte" desses periódicos. O maior número dos periódicos pesquisados está localizado nas Regiões Sudeste e Centro-Oeste, onde se concentram também um maior número de cursos de Pós-Graduação na área, estes responsáveis pela disseminação da informação científica no país.

Da análise dos temas, conclui-se que, no contex to deste estudo, não há grandes coincidências dos temas identificados nos artigos dos periódicos científicos brasileiros na área da Ciência da Informação com os publicados no LISA. Pode-se afirmar que $61,6 \%$ dos trabalhos no âmbito das revistas pesquisadas estão concentrados em três diferentes temas, evidenciando-se assim que somente $38,4 \%$ dos trabalhos estão dispersos nos sete temas restantes, ou seja, há uma grande concentração de estudos em determinadas áreas enquanto outras ainda precisam ser iniciadas. A falta de ocorrência no tema 6: Prédios de Biblioteca deixa claro que no Brasil não há preocupação em relação à ambientação e que, como se vê na prática, as bibliotecas e centros de documentação e informação, são sempre localizadas em espaços inapropriados, com algumas adaptações e nunca em espaço próprio construído especificamente para ela. Mesmo com as bibliotecas virtuais e digitais em fase de ampliação, o Brasil, país de grandes diversidades regionais (econômicas, políticas e sociais) conta com certas dificuldades em função 
das condições de acesso à informação digital, devendo, assim, preocupar-se ainda com a adequação física de suas bibliotecas.

Dos 621 autores que fazem parte do universo, apenas 152 escreveram mais de um artigo (24\%), dos quais a maioria se encontra ligada de forma direta ou indireta à docência, o que já havia sido constatado em estudos anteriores. Isso leva a crer que há uma provável falta de interesse dos técnicos da área pela pesquisa científica.

A distribuição de autores por tema demonstra uma diversidade de interesse dos autores pesquisados nesse estudo em diferentes temas, causada talvez pelo número insuficiente de pesquisadores que possam cobrir a área exigindo, assim, dos pesquisadores, estudos em diferentes linhas de pesquisa.

A predominância dos artigos com autoria única que já havia sido constatada anteriormente se mantém num percentual de $73,52 \%$. Também foi constatado nesta pesquisa que é elevado o número de autores que publicaram em somente um periódico $(86,60 \%)$. Os outros $13,40 \%$, do universo de autores, escreveram entre 2 e 5 dos 6 periódicos estudados.

Pode-se, então, afirmar que os periódicos científicos na área da Ciência da Informação no Brasil, embora com todas as dificuldades apresentadas, estão cumprindo com seu objetivo de canal indispensável de comunicação científica na divulgação dos resultados e andamentos das pesquisas efetuadas pelos estudiosos da área.

Éimportante deixar registrado que a pesquisa se ateve à colocação daquilo que se queria estudar, ou seja, obter um panorama dos temas publicados nos periódicos científicos brasileiros na área da Ciência da Informação na década de 90 visando verificar as presenças temáticas nacionais, a relação dos temas brasileiros com os internacionais e a existência de núcleo de pesquisadores. E uma visão panorâmica não preenche todos os espaços o que permite uma série de variações. Sendo assim, espera-se abrir com esse trabalho, novas frentes de pesquisa como, por exemplo, a verificação de citações dos periódicos, a publicação pelos autores mais produtivos também em literatura estrangeira, a verificação de citação a esses autores no LISA, entre outros. A pesquisa científica é um ciclo que está sempre em movimento e não se esgota em si mesma.

\section{REFERÊNCIAS}

ARAujo, W.T.; CUNHA, J. Pesquisa em Biblioteconomia/Ciência da Informação: análise de produção a partir dos periódicos da área - década de 90 . In: ENANCIB, 4., 2000, Brasília. Anais... Brasília: ENANCIB, 2000.p.513.

ARENAS, J.L. et al. Una visión bibliométrica de la investigación en Bibliotecología y Ciencia de la Información de América Latina y el Caribe. Revista Especializada em Documentação Científica, v.23, n.1, p.45-53, 2000.

DUMONT, M.M.V. et al. Análise preliminar da literatura biblioteconômica brasileira. Revista da Escola de Biblioteconomia da UFMG, Belo Horizonte, v. 8 n.2, p.185-206, 1979.

FORESTI, N.A.B.; MARTINS, M.S.M. Revistas brasileiras de Biblioteconomia, Documentação e Ciência da informação: produtividade de autores no período de 1980 a 1985. Revista da Escola de Biblioteconomia da UFMG, Belo Horizonte, v.16, n.1, p. 54-71, 1987.

FORESTI, N.A.B. Contribuição das revistas brasileiras de Biblioteconomia e Ciência da Informação enquanto fonte de referência para a pesquisa. Ciência da Informação, Brasília, v.19, n.1, p.53-71, 1990.

GUIMARÃES, C.F. Visibilidade da literatura periódica brasileira em Biblioteconomia e Ciência da Informação: 1972-1981. Rio de Janeiro: 1984. Dissertação (Mestrado em Comunicação) - Departamento de Comunicação da Escola de Comunicação, Universidade Federal do Rio de Janeiro, 1984.

LIBRARY Information Science Abstracts. Available from: <http://200.219.29.133:8590/?.nextform=history. htm\&sp.usernumber.p=351945> . Acess: 15 jan. 2001.

CONSELHO Nacional de Desenvolvimento Científico e Tecnológico (CNPq), Ministério de Ciência e Tecnologia. Disponível em: <http://www.cnpq.br/bolsas/pesq-cientifica/projetos-individuais.htm>. Acesso em: 2002.

MIRANDA, A. Revistas especializadas brasileiras em biblioteconomia e ciência da informação com ênfase na ABDF. Boletim ABDF: Nova Série, Brasília, v.4, n.4, p.30-42, 1981. 
MUELLER, S.P.M.; CAMPELLO, B.S.; DIAS, E.J.W. Disseminação da pesquisa em Ciência da Informação e Biblioteconomia no Brasil. Ciência da Informação, Brasília, v.25, n.3, 1996.

NEVES, F.I.; MELO, M.G.L. Revistas brasileiras de Biblioteconomia e documentação na década de 70. In: CONGRESSOLATINO-AMERICANODE BIBLIOTECONOMIA E DOCUMENTAÇÃO, 1., Salvador, 1980. Anais... Brasília: CAPES, 1980. v.1, p. 419-434.

OHIRA, M.L.; SOBRIO, M.L.L.N.; PRADO, N.S. Periódicos brasileiros especializados em Biblioteconomia e Ciência da Informação: evolução. Encontros Bibli, n.10, out. 2000. Disponível em: <http://www.sced.ufsc.br/ bibliote/encontro/bibli10/lurdinha.htm >. Acesso em: 21 nov. 2000.

PITTELLA, M.C. Análise de citação de periódicos brasileiros de biblioteconomia: 1972-1982. Revista da Escola de Biblioteconomia da UFMG, Belo Horizonte, v.20, n.2, p.191-217, 1991.

TEIXEIRA, S.K.S. Temática das dissertações defendidas no curso de mestrado em Biblioteconomia e Documentação da Universidade de Brasília: 1980-1995. Brasília: 1997. 135f. Dissertação (Mestrado em Ciência da Informação) - Universidade de Brasília. 


\section{ANEXOS}

\section{ANEXO 1}

TABELA DE ASSUNTOS DOS PERIÓDICOS NA ÁREA DA CIÊNCIA DA INFORMAÇÃO

\begin{tabular}{|c|c|c|}
\hline Código & Assuntos gerais e específicos & Tradução do assunto principal \\
\hline 1 & $\begin{array}{l}\text { Ciência da Informação, Biblioteconomia e } \\
\text { Documentação (generalidades) } \\
\text { Aspectos filosóficos } \\
\text { Aspectos históricos } \\
\text { Aspectos sociais } \\
\text { Biblioteca e sociedade } \\
\text { Biblioteconomia comparada } \\
\text { Biblioterapia } \\
\text { Conceitos } \\
\text { Conhecimento } \\
\text { Direitos autorais } \\
\text { Discursos } \\
\text { Epistemologia e questões da ciência } \\
\text { Estudo comparado (metodologia) } \\
\text { Evolução histórica } \\
\text { História do livro } \\
\text { Indústria da informação } \\
\text { Indústria editorial } \\
\text { Informação e sociedade } \\
\text { Interdisciplinaridade } \\
\text { Objetivos da ciência } \\
\text { Organização do conhecimento } \\
\text { Paradigmas } \\
\text { Reuniões e palestras }\end{array}$ & Information Science, Library Science and Documentation \\
\hline 2 & $\begin{array}{l}\text { Ensino, Atividade Profissional e Pesquisa } \\
\text { Objetivos educacionais } \\
\text { Programa de ensino } \\
\text { Metodologia e programa de ensino } \\
\text { Atividade de pesquisa } \\
\text { Teses e dissertações } \\
\text { Formação profissional } \\
\text { Profissional da informação } \\
\text { Auto-imagem profissional } \\
\text { Biografia do bibliotecário }\end{array}$ & Teaching of Library Science Librarian \\
\hline 3 & $\begin{array}{l}\text { Organização e Gerência de Atividades de In- } \\
\text { formação, de Bibliotecas e Centros de Pesquisa } \\
\text { Consultorias } \\
\text { Padrões de infra-estrutura } \\
\text { Gestão de qualidade } \\
\text { Comportamento gerencial } \\
\text { Estilos gerenciais } \\
\text { Motivação } \\
\text { Tomada de decisão }\end{array}$ & Management of Information Activities \\
\hline
\end{tabular}


Continuação

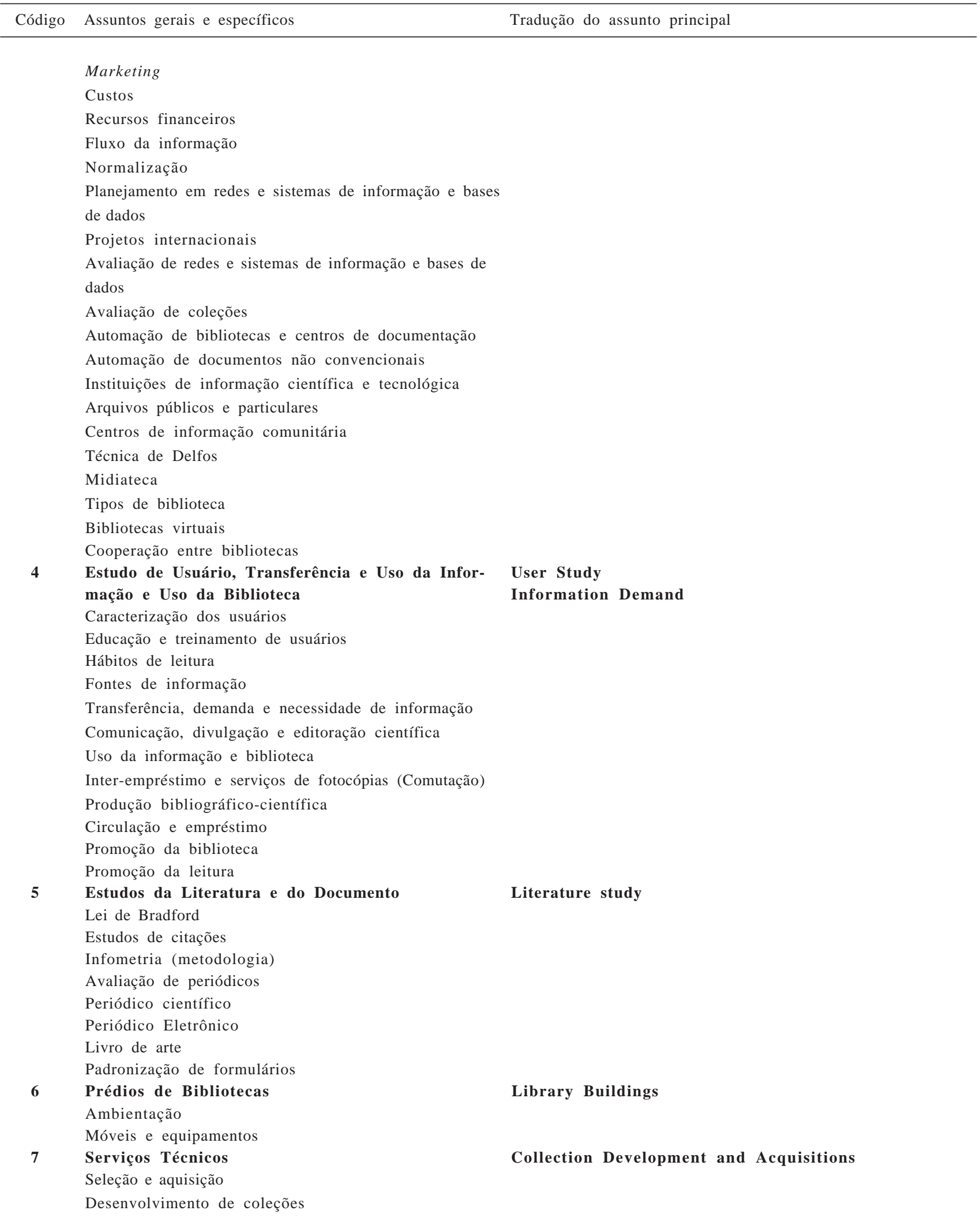


Continuação

Código Assuntos gerais e específicos

Preservação e conservação

Retirada e descarte

Segurança (proteção do acervo)

8

Tradução do assunto principal

Entrada, Tratamento, Armazenamento, Recupera-

ção e Disseminação da Informação

Depósito legal

Controle bibliográfico

Descrição bibliográfica

Referência bibliográfica

Formatos bibliográficos

Serviço de referência

Catálogos

Bibliografias

Intercâmbio de registros automatizados

Sistemas cooperativos

Indexação manual e automática (aspectos gerais e teóricos)

Tesauros (aspectos gerais e teóricos)

Elaboração de resumos

Hipermídia

Hipertexto

Videotexto

Tecnologia da informação

Teletex, Correio eletrônico, Quadro de avisos

eletrônicos

Base de dados em linha

\section{CD-ROM}

Inteligência artificial

Código de barras

Análise automática de textos

Sistemas especialistas

Redes e sistemas de informação

Disponibilidade e acessibilidade

Recuperação da informação (aspectos gerais e teóricos,

buscas)

DSI (avaliação)

Sistemas de classificação

Esquemas de palavras-chave

Sistemas de informação especializados

- Informação africanista

- Informação agrícola

- Informação ambiental

- Informação científica e tecnológica

- Informação em ciências sociais e humanas

- Informação para negócios

- Informação tecnológica

9 Política de Informação e Política Científica e

Tecnológica

Política de informação

Política científica e tecnológica

\section{Technical Processes}

Policy of Information

Scientifical and Technological Police 
Continuação

$\begin{array}{ll}\text { Código } & \text { Assuntos gerais e específicos } \\ & \text { Economia da informação do assunto principal } \\ \text { Transferência de tecnologia } & \\ \text { Tecnologia de comunicação } & \text { MERCOSUL } \\ \mathbf{1 0} \quad \text { Outros Assuntos Correlatos ou Adicionais } \\ \text { AIDS (epidemiologia) } \\ \text { Ciência da terminologia } \\ \text { Cultura brasileira (aspectos sociais) } \\ \text { Holografia } \\ \text { Informática } \\ \text { Lingüística } \\ \text { Meios de comunicação } \\ \text { Parque tecnológico } \\ \text { Política do ambiente } \\ \text { Relações públicas } \\ \text { Semiologia e semiótica } \\ \text { Traduções (aspectos teóricos e técnicos) } \\ \text { Universidades }\end{array}$

Fonte: Teixeira (1997) adaptada. 


\section{ANEXO 2}

AUTORES QUE PUBLICARAM EM MAIS DE UM PERIÓDICO (1990-1999)

\begin{tabular}{|c|c|c|c|c|c|c|}
\hline \multirow[b]{2}{*}{ Autor } & \multicolumn{6}{|c|}{ Periódico } \\
\hline & $\begin{array}{l}\text { Ciência da } \\
\text { Informação }\end{array}$ & $\begin{array}{l}\text { Informação } \\
\text { e Sociedade }\end{array}$ & Perspectivas & $\begin{array}{c}\text { Revista de } \\
\text { Biblioteconomia } \\
\text { de Brasília }\end{array}$ & $\begin{array}{l}\text { Revista da } \\
\text { UFMG }\end{array}$ & Transinformação \\
\hline 1. ALVARENGA, Lídia & $\mathrm{x}$ & & $\mathrm{x}$ & & $\mathrm{x}$ & \\
\hline 2. ALVES, Maria D. & & & $\mathrm{X}$ & & & $\mathrm{x}$ \\
\hline 3. AMARAL, Sueli A. & $\mathrm{x}$ & & $\mathrm{X}$ & $\mathrm{X}$ & & \\
\hline 4. ARAGÃO, Esmeralda M. & $\mathrm{x}$ & & & & & $\mathrm{x}$ \\
\hline 5. ARAÚJO, Eliany A. & $\mathrm{x}$ & $\mathrm{x}$ & & & & \\
\hline 6. AUN, Marta P. & $\mathrm{x}$ & & & & $\mathrm{x}$ & \\
\hline 7. BARBOSA, Ricardo R. & $\mathrm{x}$ & & $\mathrm{X}$ & & $\mathrm{x}$ & \\
\hline 8. BARRETO, Aldo A. & $\mathrm{x}$ & & & $\mathrm{x}$ & & \\
\hline 9. BARROS, Antônio T. & & & & $\mathrm{x}$ & & $\mathrm{x}$ \\
\hline 10. BETTIOL, Eugênia M. & $\mathrm{x}$ & & & $\mathrm{X}$ & & \\
\hline 11. BORGES, Mônica E.N & $\mathrm{x}$ & & $\mathrm{x}$ & & & \\
\hline 12. BUFREM, Leilah S. & & & & & $\mathrm{x}$ & $\mathrm{x}$ \\
\hline 13. CALDEIRA, Paulo T. & $\mathrm{x}$ & & $\mathrm{x}$ & & $\mathrm{x}$ & \\
\hline 14. CAMPELO, Bernadete S. & $\mathrm{x}$ & & $\mathrm{x}$ & $\mathrm{x}$ & $\mathrm{x}$ & \\
\hline 15. CAMPOS, Estela M. & & & & & $\mathrm{x}$ & $\mathrm{x}$ \\
\hline 16. CAMPOS, Maria L.A. & $\mathrm{x}$ & & & & $\mathrm{x}$ & \\
\hline 17. CARDOSO, Ana M.P. & & $\mathrm{x}$ & $\mathrm{x}$ & & $\mathrm{x}$ & \\
\hline 18. CAVAN, Michael M. & $\mathrm{x}$ & & & $\mathrm{x}$ & & \\
\hline 19. COSTA, Sely M.S. & & & & $\mathrm{x}$ & & $\mathrm{x}$ \\
\hline 20. CUNHA, Mirian V. & & & & $\mathrm{x}$ & $\mathrm{x}$ & \\
\hline 21. DANTAS, Marcos & $\mathrm{x}$ & & & & & $\mathrm{x}$ \\
\hline 22. DI CHIARA, Ivone G. & & & $\mathrm{X}$ & & $\mathrm{X}$ & \\
\hline 23. DIAS, Eduardo J.W. & $\mathrm{x}$ & & $\mathrm{x}$ & & $\mathrm{x}$ & \\
\hline 24. FIGUEIREDO, Nice M. & $\mathrm{x}$ & & $\mathrm{x}$ & & $\mathrm{x}$ & \\
\hline 25. FRANÇA, Ricardo O. & & & $\mathrm{x}$ & & $\mathrm{x}$ & \\
\hline 26. FREIRE, Isa M. & $\mathrm{x}$ & & $\mathrm{x}$ & & & $\mathrm{x}$ \\
\hline 27. FURNIVAL, Ariadne C. & $\mathrm{x}$ & & $\mathrm{x}$ & & & \\
\hline 28. GARCIA, Joana C.R. & $\mathrm{x}$ & $\mathrm{x}$ & & & & \\
\hline 29. GARDINI, Marília J.A. & & & & $\mathrm{x}$ & $\mathrm{x}$ & \\
\hline 30. GIACOMETTI, Maria M. & $\mathrm{x}$ & & & & & $\mathrm{x}$ \\
\hline 31. GONTOW, Rejane & $\mathrm{x}$ & & & & & $\mathrm{x}$ \\
\hline 32. KLAES, Rejane R. & $\mathrm{X}$ & & & $\mathrm{x}$ & & \\
\hline 33. KOBASHI, Nair Y. & $\mathrm{x}$ & & & $\mathrm{x}$ & & \\
\hline 34. KREMER, Jeannette M. & & & $\mathrm{x}$ & $\mathrm{x}$ & $\mathrm{x}$ & \\
\hline 35. KURAMOTO, Hélio & $\mathrm{x}$ & & & $\mathrm{x}$ & & \\
\hline 36. LIMA, Gercina Â.B.O. & $\mathrm{x}$ & & $\mathrm{X}$ & & $\mathrm{x}$ & \\
\hline 37. LUCAS, Clarinda R. & $\mathrm{x}$ & & & & & $\mathrm{x}$ \\
\hline 38. MARANON, Eduardo I.M. & $\mathrm{x}$ & & & & $\mathrm{x}$ & $\mathrm{x}$ \\
\hline 39. MARCHIORI, Patrícia Z. & $\mathrm{x}$ & & & $\mathrm{x}$ & & $\mathrm{x}$ \\
\hline 40. MARCONDES, Carlos H. & & & & & $\mathrm{x}$ & $\mathrm{x}$ \\
\hline 41. MARTELETO, Regina M. & $\mathrm{x}$ & & & & $\mathrm{X}$ & \\
\hline 42. MARTUCCI, Elisabeth M. & & & $\mathrm{X}$ & $\mathrm{x}$ & $\mathrm{x}$ & $\mathrm{x}$ \\
\hline 43. MELO, Denise G.P. & $\mathrm{x}$ & $\mathrm{x}$ & & & & \\
\hline
\end{tabular}


Continuação

\begin{tabular}{|c|c|c|c|c|c|c|}
\hline \multirow[b]{2}{*}{ Autor } & \multicolumn{6}{|c|}{ Periódico } \\
\hline & $\begin{array}{l}\text { Ciência da } \\
\text { Informação }\end{array}$ & $\begin{array}{l}\text { Informação } \\
\text { e Sociedade }\end{array}$ & Perspectivas & $\begin{array}{c}\text { Revista de } \\
\text { Biblioteconomia } \\
\text { de Brasília }\end{array}$ & $\begin{array}{l}\text { Revista da } \\
\text { UFMG }\end{array}$ & Transinformação \\
\hline 44. MENEZES, Estera M. & $\mathrm{X}$ & & & & & $\mathrm{X}$ \\
\hline 45. MOSTAFA, Solange P.I. & $\mathrm{X}$ & & & & $\mathrm{X}$ & $\mathrm{X}$ \\
\hline 46. MUELLER, Suzana P.M. & $\mathrm{X}$ & & & $\mathrm{X}$ & $\mathrm{X}$ & \\
\hline 47. NASTRI, Rosemeire M. & & & $X$ & & $\mathrm{X}$ & $X$ \\
\hline 48. NAVES, Madalena M.L. & $\mathrm{X}$ & & $\mathrm{X}$ & $\mathrm{X}$ & & \\
\hline 49. NEGRÃO, May B. & $\mathrm{X}$ & & & $\mathrm{X}$ & & \\
\hline 50. NEHMY, Rosa M.Q. & $\mathrm{X}$ & & $\mathrm{X}$ & & & \\
\hline 51. NEVES, Jorge T.C. & & & $\mathrm{X}$ & & & $\mathrm{X}$ \\
\hline 52. NORONHA, Daisy P. & $\mathrm{X}$ & & & & & $\mathrm{X}$ \\
\hline 53. OCTAVIANO, Vera L.C. & $\mathrm{X}$ & & & & & $\mathrm{X}$ \\
\hline 54. OLIVEIRA, Margarida P. & $\mathrm{X}$ & & & & & $\mathrm{X}$ \\
\hline 55. OLIVEIRA, Maria J. & $\mathrm{X}$ & & & $\mathrm{X}$ & & \\
\hline 56. OLIVEIRA, Nirlei M. & & & $X$ & & & $\mathrm{X}$ \\
\hline 57. OLIVEIRA, Silas M. & & & & $\mathrm{X}$ & & $\mathrm{X}$ \\
\hline 58. PACHECO, Márcia & $\mathrm{X}$ & & & & & $\mathrm{X}$ \\
\hline 59. PAIM, Isís & $\mathrm{X}$ & & $\mathrm{X}$ & & $\mathrm{X}$ & \\
\hline 60. PASSARELLI, Brasiliana & $\mathrm{X}$ & & & $\mathrm{X}$ & & \\
\hline 61. PEREIRA, Maria N.F. & $\mathrm{X}$ & & & $\mathrm{X}$ & & \\
\hline 62. PINHEIRO, Lena V.R. & $\mathrm{X}$ & & & $\mathrm{X}$ & & \\
\hline 63. PITELLA, Mônica C. & & & $\mathrm{X}$ & & $\mathrm{X}$ & \\
\hline 64. POBLACIÓN, Dinah A. & $\mathrm{X}$ & & & & & $\mathrm{X}$ \\
\hline 65. PONTES, Cecília C.C. & $\mathrm{X}$ & & & & & $\mathrm{X}$ \\
\hline 66. PRAZERES, Yara M.P.C. & & & $\mathrm{X}$ & & $\mathrm{X}$ & \\
\hline 67. REIS, Alcenir S. & & & $\mathrm{X}$ & & $\mathrm{X}$ & \\
\hline 68. ROBREDO, Jaime & $\mathrm{X}$ & & & & & $\mathrm{X}$ \\
\hline 69. SANTOS, Raimundo N.M. & $\mathrm{X}$ & & & $\mathrm{X}$ & & \\
\hline 70. SARACEVIC, Tefko & $\mathrm{X}$ & & & & & \\
\hline 71. SCHMIDT, Susana & $\mathrm{X}$ & & & $\mathrm{X}$ & & \\
\hline 72. SOUZA, Francisco C. & $\mathrm{X}$ & $\mathrm{X}$ & & $\mathrm{X}$ & & \\
\hline 73. SOUZA, Terezinha C. & $\mathrm{X}$ & & $\mathrm{X}$ & & & \\
\hline 74. STUMPF, Ida R.C. & $\mathrm{X}$ & & & $\mathrm{X}$ & & $\mathrm{X}$ \\
\hline 75. TARAPANOFF, Kira & $\mathrm{X}$ & & & $\mathrm{X}$ & & $\mathrm{X}$ \\
\hline 76. TARGINO, Maria G. & $\mathrm{X}$ & $\mathrm{X}$ & & $\mathrm{X}$ & $\mathrm{X}$ & $\mathrm{X}$ \\
\hline 77. VALLS, Valéria M. & $\mathrm{X}$ & & $\mathrm{X}$ & & & \\
\hline 78. VERGUEIRO, Waldomiro C.S. & $\mathrm{X}$ & & $\mathrm{X}$ & & $\mathrm{X}$ & \\
\hline 79. VICENTE, Gilmar & & & $\mathrm{X}$ & & & $\mathrm{X}$ \\
\hline 80. VIEIRA, Ana S. & $\mathrm{X}$ & & & & $\mathrm{X}$ & \\
\hline 81. VILAN FILHO, Jayme L. & $\mathrm{X}$ & & & $\mathrm{X}$ & & \\
\hline 82. WITTER, Geraldina P. & & & & $\mathrm{X}$ & & $\mathrm{X}$ \\
\hline
\end{tabular}

Fonte: Revistas Ciência da Informação, Informação e Sociedade, Perspectivas, Revista de Biblioteconomia de Brasília, Revista da UFMG, Transinformação (1990-1999). 
